\title{
The effect of fennel essential oil and trans-anethole on antibacterial activity of mupirocin against Staphylococcus aureus isolated from asymptomatic carriers
}

\author{
Paweł Kwiatkowski ${ }^{1}$, Agata Pruss ${ }^{1}$, Helena Masiuk ${ }^{1}$, Magdalena Mnichowska-Polanowska', Maciej Kaczmarek², \\ Stefania Giedrys-Kalemba', Barbara Dołęgowska', Hanna Zielińska-Bliźniewska³ ${ }^{3}$ Jurek Olszewski ${ }^{4}$, \\ Monika Sienkiewicz ${ }^{3}$
}

${ }^{1}$ Department of Microbiology, Immunology and Laboratory Medicine, Pomeranian Medical University, Szczecin, Poland 2Department of Experimental and Clinical Pharmacology, Pomeranian Medical University, Szczecin, Poland ${ }^{3}$ Department of Allergology and Respiratory Rehabilitation, $2^{\text {nd }}$ Chair of Otolaryngology, Medical University of Lodz, Lodz, Poland ${ }^{4}$ Department of Otolaryngology and Laryngological Oncology, $2^{\text {nd }}$ Chair of Otolaryngology, Medical University of Lodz, Lodz, Poland

Adv Dermatol Allergol 2019; XXXVI (3): 308-314 DOI: https://doi.org/10.5114/ada.2018.76425

\begin{abstract}
Introduction: Staphylococcus aureus constitutes the most pathogenic species within the staphylococcal genus. Humans remain the major reservoirs of this pathogen which colonizes mostly anterior nares of healthy individuals. Aim: To investigate the effect of fennel essential oil (FEO) and trans-anethole ( $t A)$ on antibacterial activity of mupirocin (MUP) against $S$. aureus strains isolated from asymptomatic carriers.

Material and methods: The content of the FEO was analysed with use of the GC-MS method. The research done on $43 \mathrm{~S}$. aureus isolates with different resistance patterns, obtained from nasal vestibule. Antibacterial activity of MUP in combination with FEO or tA was examined using the agar dilution method and E-test method. The data analysis was done with the Pearson's $\chi^{2}$ test.

Results: The chemical composition of FEO was consistent with the European Pharmacopoeia (EP) for the main constituent - tA (77.9\%) according to the EP recommendations. Macrolide-lincosamide-streptogramin B resistance phenotype was prevalent among $39.5 \%$ of $S$. aureus isolates. FEO concentrations of $2.0 \%$ and $2.5 \%$ revealed antibacterial activity against $76.7 \%$ of isolates, whereas $t A$ inhibited S. aureus growth at concentrations $>4.0 \%$. The MIC values for MUP combined with FEO as well as for MUP combined with tA were $<0.064 \mu \mathrm{g} / \mathrm{ml}$ for $79.1 \%$ and $86.0 \%$ of $S$. aureus isolates, respectively.

Conclusions: Our experiment revealed FEO and tA influence on MUP effectiveness. The combination of MUP with FEO as well as MUP with $t A$ are worth considering to implement in $S$. aureus eradication procedures. These findings will be useful in designing efficient antistaphylococcal agents which can limit the emergence of antibiotic resistance.
\end{abstract}

Key words: Staphylococcus aureus, mupirocin, fennel essential oil, trans-anethole.

\section{Introduction}

An extensive transmission of Staphylococcus aureus between humans is known to be significantly attributed to asymptomatic carriers. The percentage of persistent carriers of $S$. aureus in anterior nares is reckoned to be about $20 \%$, whereas the transient nasal carriers and non-carriers account for $60 \%$ and $20 \%$, respectively. The persistent carrier-state is being more frequently observed among young children than adults; however the incidence of S. aureus carriage among listed groups undergoes numerous changes in individuals aged between 10 and 20 [1]. Higher rates of nasal colonization are observed in hospital staff (around 44\%), which doubtlessly poses a great risk of $S$. aureus transmission to hospitalized patients [2, 3].

The carriage of $S$. aureus among medical staff represents an outstanding problem because these can transfer multidrug staphylococcal strains to medical students, other medical staff, and, most importantly, to hospitalized patients. It is estimated that the nasal carriage of S. aureus among medical staff ranges between $16.8 \%$ and $56.1 \%$ [1]. Nasal colonization with $S$. aureus is a risk factor for endogenous staphylococcal infection, especially in patients undergoing surgical procedures with simultaneously reduced immunity. Thus, the eradication is needed in carriers to reduce the risk of infection.

Address for correspondence: Paweł Kwiatkowski MSc Eng, Department of Microbiology, Immunology and Laboratory Medicine, Pomeranian Medical University, 72 Powstancow Wielkopolskich St, 70-111 Szczecin, Poland, phone: +48 914661652 , fax: +48 914661659 , e-mail: pawel.kwiatkowski@pum.edu.pl

Received: 21.02.2018, accepted: 1.04.2018. 
Available guidelines recommend a topical application of mupirocin (MUP) on mucosal membranes of anterior nares to eradicate the $S$. aureus colonization (methicillinsensitive S. aureus - MSSA and methicillin-resistant S. aureus - MRSA). Due to a high incidence of re-colonization in individuals treated with MUP, it is recommended to use the ointment for at least several weeks [4]. The worldwide increase in resistance to MUP among $S$. aureus explains the need to evaluate the susceptibility to MUP of each S. aureus isolate as well as the necessity to search for new treatment options such as application of essential oils which express good antimicrobial properties [5].

Foeniculum vulgare Mill. (fennel), biennial plant belonging to the Apiaceae family has been easily adapted to many geographic regions [6]. Due to its unique taste, aroma and antimicrobial action, fennel is used in food and cosmetic industry [7]. Fennel is also used to treat gastrointestinal and respiratory tracts disorders. It is known to cause intestinal smooth muscles relaxation and to dissolve bronchial secretion, thus to facilitate easier expectoration. Moreover, the fennel seed extract promotes milk supply in breasts of feeding mothers, assuages period pains, increases sex drive and facilitates birth. Available studies reveal that fennel essential oil (FEO) displays antimicrobial, antioxidant, anti-inflammatory, anti-cancer, anti-spasmodic, anti-coagulant, anti-diabetic, cholagogue, diuretic, anti-hypertensive, hepatoprotective and acaricidal properties [8-10].

FEO is composed mostly of trans-anethole (tA) and fenchone followed by camphene, estragole, limonene, $\alpha$-pinene, $\beta$-pinene, $\beta$-myrcene, camfor, 3 -carene, $\alpha$-phellandrene and cis-anethole [9].

tA ( $p$-metoxypropenylobenzene) is an organic compound, a by-product of terpene synthesis. It constitutes the major ingredient of many essential oils - anise (Pimpinella anisum L.), star anise (Illicium verum Hook. f.) and fennel (F. vulgare Mill.). tA is widely used in food industry, but also in cosmetic and health-care industries $[11,12]$. Existing evidence proves its antimicrobial, insecticidal, larvicidal and antioxidative properties [7, 13].

\section{Aim}

The aim of the study was to evaluate the influence of FEO and tA on antibacterial activity of MUP against S. aureus strains isolated from asymptomatic carriers.

\section{Material and methods}

\section{Strains and their susceptibility to antimicrobial agents}

Staphylococcus aureus strains were isolated from medical faculties' students during the 2-years study, in the years 2014-2015 at Department of Microbiology, Immunology and Laboratory Medicine of Pomeranian Medical University in Szczecin, Poland. During the study period,
43 bacterial strains from anterior nares swabs have been collected and routinely all were identified with $S$. aureus screening assay. The specimens were cultivated on Columbia agar with $5 \%$ sheep blood (bioMérieux, Poland), identified by colony morphology, tube coagulase test (Biomed, Poland), Staph-Kit test (bioMérieux, Poland) and biochemical test GP Vitek 2 Compact (bioMérieux, Poland). S. aureus ATCC $^{\circledR} 29213^{\text {TM }}$ strain was used to determine the optimal concentration of FEO and $t A$ in combination with increasing (E-test method) concentrations of MUP (bioMérieux, Poland).

The susceptibility to ciprofloxacin, co-trimoxazole and MUP of S. aureus isolates was assessed via disc diffusion method and interpreted according to the European Committee on Antimicrobial Susceptibility Testing (EUCAST) recommendations. MRSA phenotype was determined using cefoxitin. The presence of macrolide-lincosamidestreptogramin $B\left(M L S_{B}\right)$ resistance phenotype was determined using erythromycin and clindamycin in accordance with Clinical and Laboratory Standards Institute (CLSI) recommendations [14-17]. Antibiotic discs were provided by Diag-Med, Poland.

\section{FEO and $t \mathrm{~A}$ origin}

FEO purchased from Pollena-Aroma (Poland) was used in this study. The essential oil was analysed by gas chromatography-mass spectrometry (GC-MS) at the Faculty of Chemical Technology and Engineering, West Pomeranian University of Technology in Szczecin. The main constituent of tested essential oil was tA (77.9\%) followed by fenchone (12.8\%), $\alpha$-pinene (3.8\%), estragole (2.3\%), limonene (2.1\%) [14].

tA with a purity of not less than $99.0 \%$ was obtained from Sigma-Aldrich, Germany.

\section{Susceptibility of S. aureus to FEO and tA}

The agar dilution method mirrored the one approved by CLSI [18] with the following modification: a final concentration of $1.0 \%(\mathrm{v} / \mathrm{v})$ Tween $^{\oplus} 80$ (Sigma-Aldrich, Germany) was incorporated into Mueller-Hinton Agar (MHA) (Sigma-Aldrich, Germany) after autoclaving in order to enhance FEO and tA solubility. FEO and tA at various concentrations $(0.1 \%, 0.25 \%, 0.5 \%, 0.75 \%, 1.0 \%, 1.5 \%, 2.0 \%$, $2.5 \%, 3.0 \%, 3.5 \%, 4.0 \%)(\mathrm{v} / \mathrm{v})$ were independently transferred into MHA with $1.0 \%(\mathrm{v} / \mathrm{V})$ Tween $^{\oplus} 80$. Plates were dried at $37^{\circ} \mathrm{C}$ for $30 \mathrm{~min}$ prior to inoculation of each them with suspension containing approximately $10^{4} \mathrm{CFU} / \mathrm{ml}$ of particular S. aureus isolates, using a multichannel pipette. MHA with $1.0 \%(\mathrm{v} / \mathrm{v})$ Tween $^{\oplus} 80$ without FEO or tA was used as a positive growth control for $S$. aureus. Minimal inhibitory concentration (MIC) of each tested ingredient was determined after $18 \mathrm{~h}$ incubation and recognized as the lowest concentration of oil which completely inhibited the visible growth of S. aureus on MHA plate. According 
to CLSI recommendations, a single colony or a faint haze caused by the inoculum was disregarded [18].

\section{Determination of the optimal concentration of FEO and $t \mathrm{~A}$ in combination with MUP}

Determination of the optimal FEO and tA concentration enhancing antibacterial activity of MUP was assessed using ATCC $^{\oplus} 29213^{\mathrm{TM}}$ strain of S. aureus. The optimization was performed using the agar dilution method and E-test method. For screening of the optimal concentrations of FEO and tA enhancing antibacterial activity of MUP, various concentrations $(0.1 \%, 0.25 \%, 0.5 \%, 0.75 \%$, $1.0 \%, 1.5 \%, 2.0 \%, 2.5 \%, 3.0 \%, 3.5 \%, 4.0 \%)(\mathrm{v} / \mathrm{v})$ of these chemicals were distributed into MHA with $1.0 \%(\mathrm{v} / \mathrm{v})$ Tween ${ }^{\circledast} 80$. Plates were dried for $30 \mathrm{~min}$ at $37^{\circ} \mathrm{C}$ prior to inoculation with sterile cotton swab containing approximately $10^{8} \mathrm{CFU} / \mathrm{ml}$ of microorganism. Then, the activity of the antibiotic was studied by applying a plastic test strip (E-test) which consisted a predefined, continuous and exponential gradient of MUP on the surface of MHA plates (with and without chemicals - control). The plates were incubated for $18 \mathrm{~h}$ at $37^{\circ} \mathrm{C}$ in aerobic conditions. After incubation, the MIC of MUP were calculated. Each test was performed with two replicates.

\section{Antibacterial activity of MUP in combination with FEO or tA}

Antibacterial activity of MUP in combination with FEO or tA was examined using the agar dilution method and E-test method in comparison with optimal concentrations revealed by optimization assay. For the screening of antibacterial activity of MUP combined with chemicals, the optimal concentration $(\mathrm{v} / \mathrm{v})$ of the chemicals was distributed in MHA with $1.0 \%(\mathrm{v} / \mathrm{v})$ Tween $^{\circledR}$ 80. Plates were dried for $30 \mathrm{~min}$ at $37^{\circ} \mathrm{C}$ prior to inoculation with $10^{8} \mathrm{CFU} / \mathrm{ml}$ of bacterial suspension. The activity of the antibiotic was studied by applying a plastic test strip (E-test) with a continuous and exponential gradient of MUP on the surface of MHA plates (with and without chemicals - control). The plates were incubated for $18 \mathrm{~h}$ at $37^{\circ} \mathrm{C}$ in aerobic conditions. The MIC values were calculated.

\section{Ethical statement}

The study was approved (KB-0012/04/01/14) by the Ethics Committee of the Pomeranian Medical University of Szczecin, Poland.

\section{Statistical analysis}

Comparison of the study data was done using the Pearson's $\chi^{2}$ test. Statistical analyses were performed using the Statistica programme version 12.5. A $p$-value $<0.05$ was considered statistically significant.

\section{Results}

\section{Screening of bacterial susceptibility to antibiotics}

All S. aureus strains isolated from the asymptomatic carriers, when tested by disc diffusion method, were susceptible to MUP and to cefoxitin (confirmed MSSA phenotype), whereas one of them showed resistance to ciprofloxacin and another one to co-trimoxazole. The resistance to erythromycin and clindamycin was observed in $39.5 \%$ of the isolates. The presence of inducible $\mathrm{MLS}_{B}$ and constitutive $M_{L S}$ resistance phenotypes was indicated in $34.9 \%$ and $4.7 \%$ of the strains, respectively. None of the isolates had $\mathrm{MS}_{\mathrm{B}}$ resistance phenotype. S. aureus ATCC $^{\circledR} 29213^{\text {TM }}$ was susceptible to all antibiotics.

\section{Determination of MICs of FEO and $t \mathrm{~A}$}

Our investigation showed that FEO in concentrations of $2.0 \%$ and $2.5 \%$, represented an inhibiting activity against $76.7 \%(p<0.0001)$ of $S$. aureus isolates. tA had much weaker inhibitory activity against staphylococci. An equal number of tested isolates (74.4\%) were susceptible to $t A$ at a concentration higher than $4.0 \%(p<0.0001)$ (Table 1). The minimal concentrations of FEO and tA inhibiting growth of S. aureus ATCC ${ }^{\oplus} 29213^{\mathrm{TM}}$ cells were $2.0 \%$ and $>4.0 \%$, respectively. It was also observed that the addition of $1 \%$ Tween ${ }^{\oplus} 80$ has no impact on growth inhibition of reference strain and isolates.

\section{Optimization of FEO and tA concentrations against S. aureus ATCC $^{\circledR} 29213^{\text {TM }}$}

The optimal concentrations of FEO or tA, enhancing the activity of MUP against reference $S$. aureus strain were $0.75 \%$ for $\mathrm{FEO}$ and $1.5 \%$ for $\mathrm{tA}$. It was observed that the addition of FEO and tA to MHA with $1 \%$ Tween $^{\oplus} 80$ decreased the MIC of MUP from $0.25 \mu \mathrm{g} / \mathrm{ml}$ to $\leq 0.064$ $\mu \mathrm{g} / \mathrm{ml}$ (Figure 1). The minimal concentration of MUP combined with $0.75 \%$ FEO and $1.5 \%$ tA (which has been recognized to inhibit the growth of reference strain), was lower than $0.064 \mu \mathrm{g} / \mathrm{ml}$ (Figure 2). It was also noted that the addition of $1 \%$ Tween ${ }^{\oplus} 80$ had no impact on the MIC value of MUP alone.

\section{Influence of FEO or tA on antibacterial activity of MUP}

A significant increase in antistaphylococcal activity of MUP combined with FEO or tA was confirmed by the agar dilution method and E-test method. The influence of FEO or tA used in combination with MUP was comparable. MICs of MUP combined with FEO and MUP combined with tA were $<0.064 \mu \mathrm{g} / \mathrm{ml}$ for $79.1 \%$ $(p<0.0001)$ and $86.0 \%(p<0.0001)$ of $S$. aureus isolates, respectively. However, MICs of MUP without FEO or tA were $0.19-0.125 \mu \mathrm{g} / \mathrm{ml}$ for $69.8 \%$ of $S$. aureus strains. The addition of FEO or tA to MHA reduced MIC values from $0.17 \pm 0.07 \mu \mathrm{g} / \mathrm{ml}$ to $0.064 \mu \mathrm{g} / \mathrm{ml}(p<0.0001)$, but 
Table 1. Minimum inhibitory concentration (MIC) of fennel essential oil (FEO), trans-anethole (tA) and mupirocin (MUP) for $S$. aureus strains isolated from the asymptomatic carriers

\begin{tabular}{|c|c|c|c|c|}
\hline $\begin{array}{r}\text { Strain } \\
\text { number }\end{array}$ & $\begin{array}{l}\text { Resistance } \\
\text { phenotype }\end{array}$ & $\begin{array}{c}\text { MIC of } \\
\text { FEO* (\%) }^{*}\end{array}$ & $\begin{array}{l}\text { MIC of } \\
t A^{*}(\%)\end{array}$ & $\begin{array}{c}\text { MIC of MUP } \\
{[\mu \mathrm{g} / \mathrm{ml}]}\end{array}$ \\
\hline 1 & - & 2.0 & 3.0 & 0.25 \\
\hline 2 & $\mathrm{iMLS}^{\mathrm{B}}$ & 2.5 & $>4.0$ & 0.19 \\
\hline 3 & iMLSB & 2.5 & $>4.0$ & 0.38 \\
\hline 4 & $\mathrm{iMLS}^{\mathrm{B}}$ & 1.5 & $>4.0$ & 0.19 \\
\hline 5 & $\mathrm{iMLS}^{\mathrm{B}}$ & 2.5 & $>4.0$ & 0.38 \\
\hline 6 & $\mathrm{iMLS}^{\mathrm{B}}$ & 2.5 & $>4.0$ & 0.125 \\
\hline 7 & $\mathrm{iMLS}^{\mathrm{B}}$ & 2.0 & $>4.0$ & 0.125 \\
\hline 8 & $\mathrm{iMLS}^{\mathrm{B}}$ & 2.0 & $>4.0$ & 0.19 \\
\hline 9 & - & 2.0 & $>4.0$ & 0.125 \\
\hline 10 & - & 2.5 & $>4.0$ & 0.25 \\
\hline 11 & - & 2.5 & $>4.0$ & 0.125 \\
\hline 12 & $\mathrm{iMLS}^{\mathrm{B}}$ & 1.5 & 4.0 & 0.125 \\
\hline 13 & $\mathrm{iMLS}^{\mathrm{B}}$ & 1.5 & $>4.0$ & 0.25 \\
\hline 14 & - & 2.5 & 4.0 & 0.25 \\
\hline 15 & - & 2.0 & $>4.0$ & 0.19 \\
\hline 16 & - & 2.5 & $>4.0$ & 0.19 \\
\hline 17 & - & 2.0 & $>4.0$ & 0.19 \\
\hline 18 & - & 2.0 & 4.0 & 0.19 \\
\hline 19 & - & 2.0 & $>4.0$ & 0.25 \\
\hline 20 & - & 2.5 & $>4.0$ & 0.094 \\
\hline 21 & - & 1.0 & $>4.0$ & 0.125 \\
\hline 22 & - & 2.5 & $>4.0$ & 0.125 \\
\hline
\end{tabular}

\begin{tabular}{|c|c|c|c|c|}
\hline $\begin{array}{r}\text { Strain } \\
\text { number }\end{array}$ & $\begin{array}{l}\text { Resistance } \\
\text { phenotype }\end{array}$ & $\begin{array}{c}\text { MIC of } \\
\text { FEO* (\%) }\end{array}$ & $\begin{array}{l}\text { MIC of } \\
t A^{*}(\%)\end{array}$ & $\begin{array}{c}\text { MIC of MUP } \\
{[\mu \mathrm{g} / \mathrm{ml}]}\end{array}$ \\
\hline 23 & - & 2.0 & $>4.0$ & 0.125 \\
\hline 24 & $\mathrm{iMLS}^{\mathrm{B}}$ & 1.0 & $>4.0$ & 0.19 \\
\hline 25 & - & 2.0 & $>4.0$ & 0.125 \\
\hline 26 & - & 2.0 & $>4.0$ & 0.094 \\
\hline 27 & - & 2.5 & $>4.0$ & 0.19 \\
\hline 28 & - & 2.5 & $>4.0$ & 0.25 \\
\hline 29 & - & 2.5 & $>4.0$ & 0.19 \\
\hline 30 & - & 1.5 & $>4.0$ & 0.25 \\
\hline 31 & - & 1.0 & $>4.0$ & 0.125 \\
\hline 32 & - & 1.5 & $>4.0$ & 0.19 \\
\hline 33 & iMLS $^{B}$ & 2.0 & $>4.0$ & 0.125 \\
\hline 34 & $\mathrm{CMLS}^{\mathrm{B}}$ & 2.5 & 3.5 & 0.125 \\
\hline 35 & $\mathrm{iMLS}^{\mathrm{B}}$ & 2.5 & 3.5 & 0.25 \\
\hline 36 & - & 1.5 & 3.5 & 0.125 \\
\hline 37 & $\mathrm{iMLS}^{\mathrm{B}}$ & 2.0 & 3.5 & 0.19 \\
\hline 38 & - & 2.0 & 3.5 & 0.125 \\
\hline 39 & $\mathrm{iMLS}^{\mathrm{B}}$ & 1.5 & $>4.0$ & 0.125 \\
\hline 40 & $\mathrm{iMLS}^{\mathrm{B}}$ & 2.0 & 3.0 & 0.125 \\
\hline 41 & $\mathrm{iMLS}^{\mathrm{B}}$ & 2.0 & 3.5 & 0.19 \\
\hline 42 & $\mathrm{CMLS}^{\mathrm{B}}$ & 2.0 & $>4.0$ & 0.094 \\
\hline 43 & $\mathrm{iMLS}^{\mathrm{B}}$ & 2.0 & $>4.0$ & 0.125 \\
\hline
\end{tabular}

$p<0.0001$. $i M L S_{B}$ - inducible macrolide-lincosamide-streptogramin B resistance phenotype, $C M L S_{B}$-constitutive macrolide-lincosamide-streptogramin $B$ resistance phenotype.

the largest number of isolates (34 and 37 strains of $S$. aureus, respectively for FEO and $t A$ ) were inhibited by a concentration lower than $0.064 \mu \mathrm{g} / \mathrm{ml}(p<0.0001)$. No correlation was found between resistance phenotypes and susceptibility towards MUP with FEO or tA combinations (Table 1).

\section{Discussion}

The anethole-dominated FEO represents one of the two reference FEO chemotypes accepted by Pharmacopoeia for medical application [19]. The content of tA in FEO ranges from 38.8\% (Albania, Yugoslavia) to $84.1 \%$ (Turkey, Albania) and is dependent on its geographical origin. The content of $t A$ in our tested FEO was close to French and Argentinean fennel oil; those contain 75.5\% and $79.9 \%$ of $t A$, respectively [20]. The European Pharmacopoeia (EP) requires minimum $60 \%$ of $t A$ in bitter $\mathrm{FEO}$ composition. The remaining needed components are as follows: $15 \%$ of fenchone, $1-10 \%$ of $\alpha$-pinene; not more than $6 \%$ of estragole and $1-5 \%$ of limonene. The chemical composition of the tested FEO was consistent with $E P$ requirements regarding $t A, \alpha$-pinene, estragole and

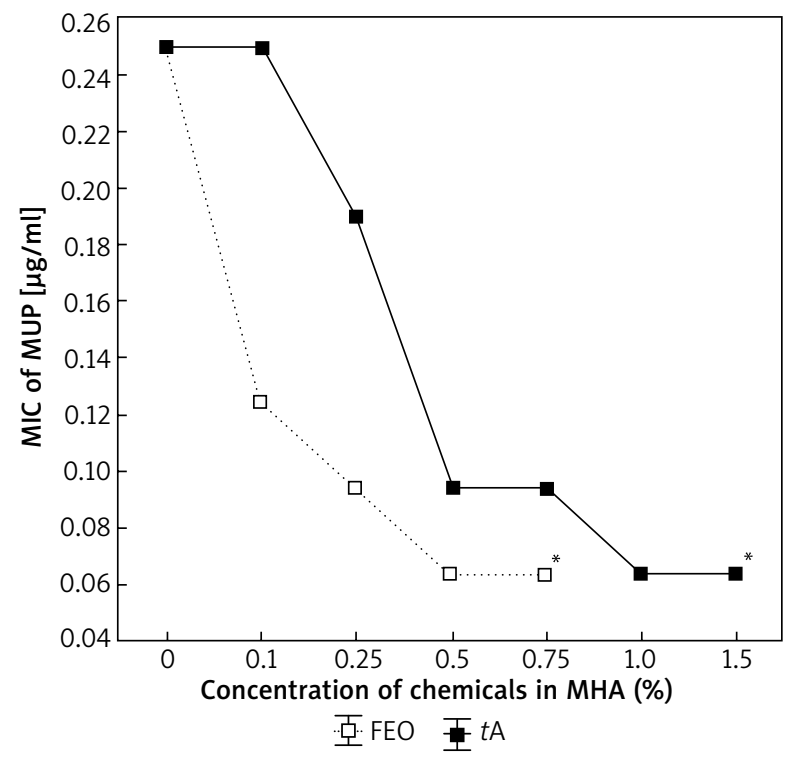

Figure 1. Determination of the optimal concentration of fennel essential oil (FEO) and trans-anethole ( $t \mathrm{~A})$ affecting the minimum inhibitory concentration (MIC) of mupirocin (MUP) against S. aureus ATCC ${ }^{\oplus} 29213^{\text {TM }}$. Concentration lower than $0.064 \mu \mathrm{g} / \mathrm{ml}\left({ }^{*}\right)$, Mueller-Hinton Agar (MHA) 

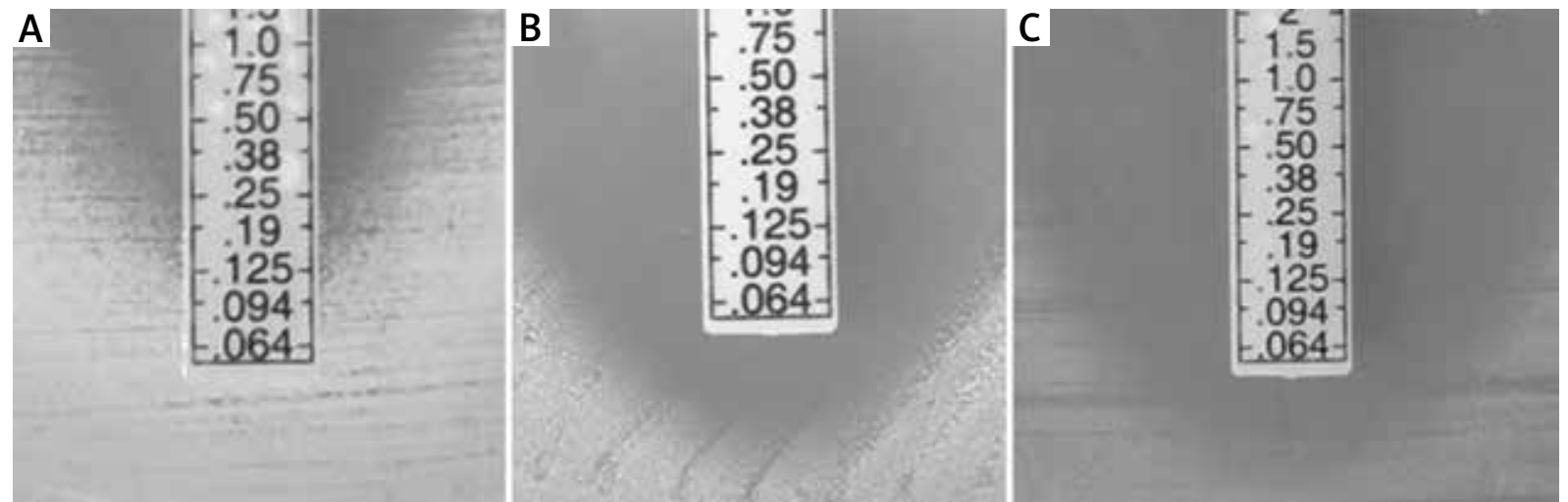

Figure 2. Minimum inhibitory concentration of mupirocin against S. aureus ATCC $® 29213^{\mathrm{TM}}$ : A - Mueller-Hinton Agar (MHA) supplemented with $1 \%(\mathrm{v} / \mathrm{v})$ Tween ${ }^{\circledR} 80$ without fennel essential oil (FEO) or trans-anethole $(t A)$, B - MHA supplemented with $1 \%(\mathrm{v} / \mathrm{v})$ Tween ${ }^{\circledR} 80$ and $0.75 \%$ FEO, C - MHA supplemented with $1 \%(\mathrm{v} / \mathrm{v})$ Tween ${ }^{\circledR} 80$ and $1.5 \%$ tA

limonene. The content of fenchone was slightly lower when compared with reference data.

According to the available data, the increase in resistance among $S$. aureus to macrolide, lincosamide and streptogramin B antibiotics is being observed [21]. The presence of $M L S_{B}$ resistance mechanism among staphylococci excludes treatment with the above-mentioned antibiotics, while it is known that erythromycin and clindamycin are commonly used in empirical treatment of Gram-positive bacteria infections. Clindamycin is an alternative therapeutic option for the treatment of staphylococcal skin and soft tissue infections, but also an alternative for patients with penicillin allergies [22]. The degree of resistance to macrolides among MRSA is still increasing, as well in MSSA, and this constitutes a significant challenge for clinicians dealing with skin infections $[23,24]$. According to the study of Yilmaz et al., the inducible $M L S_{B}$ resistance phenotype was detected in $14.8 \%$ of MSSA isolates [25]. Similar results were obtained by Prabhu et al., who reported that constitutive and inducible $M_{\text {LS }}$ resistance phenotype among MSSA occurred in $18 \%$ of isolates from different clinical samples [22]. Our results indicate that medical staff is a significant factor in the spread of pathogens resistant to macrolide, lincosamide and streptogramin B antibiotics. Therefore, there is a need for continuous monitoring of this type of bacterial resistance and implementation of new methods to prevent the spread of resistance.

In our previous study we obtained promising results and indicated that FEO was the most effective in combination with MUP [14]. Using the disc diffusion method, the increasing zone of growth inhibition of S. aureus was found. We further decided to design an exact research using the agar dilution method and $\mathrm{E}$ test method against higher number of isolates from nasal vestibule with different patterns of resistance. We studied not only the combination of FEO with MUP, but also the combination of FEO main component - tA with
MUP which, according to available data, improves cutaneous wound healing. This provides data on its oral $L_{50}$ values between 1.8-5.0 g for mice, rats and guinea pigs $[26,27]$. It can be concluded that antimicrobial activity of FEO compared with $t A$ is determined by more than one particular component, and depends on the synergistic activities of different oil constituents. This is even despite $t A$ high content. The MIC values of FEO were much lower than MICs of $t A$, ranging from 1.5 to $2.5 \%$ and from 3.0 to $>4.0 \%$, respectively. Summarizing, better results were obtained when combined FEO with MUP. The similar results were presented by Kazemi et al. in the study on antistaphylococcal activity of FEO containing mainly tA, thymol, $\alpha$-pinene, 1,8-cineole and fenchone [28]. They found that analysed FEO was more effective against human pathogenic S. aureus with $\mathrm{MIC}-0.5 \mathrm{\mu g} / \mathrm{ml}$ than the main constituent - tA itself ( $\mathrm{MIC}-4.0 \mu \mathrm{g} / \mathrm{ml})$. Only pure thymol presented comparable activity to phenolic compounds of this oil. Lachowicz et al. showed the lesser antimicrobial activity of artificially prepared main constituent mixture (linalool-estragole) compared with anise oil [29]. These results indicated that other less predominant components in anise oil most likely contribute to its antimicrobial activity.

Many scientific centres are investigating effective combinations of chemical agents as well as compounds of natural origin on decrease the resistance of microorganisms. Itaqui et al. investigated the interactions between azithromycin and terbinafine in combination with MUP in order to reduce the therapeutic doses of systemic and local treatment against Pythium insidiosum responsible for ocular, cutaneous/subcutaneous, vascular and disseminated pythiosis [30]. Sato et al. found a synergistic effect of MUP and isoflavanone (Bidwillon B) obtained from Erythrina variegata against MRSA [31]. The results showed that Bidwillon B appears to be not only a strong antistaphylococcal agent itself, but also combined with MUP, and thus can be helpful in the elimination of nasal 
and skin MRSA carriage. According to the findings of Kifer et al., some combinations of MUP and monoterpenes such as menthol, thymol, 1,8-cineole could be applied in the therapy of infections caused by $S$. aureus, including chronic rhinosinusitis [32]. In this case, the treatment is based on bacterial biofilm growth prevention. Because of the common use of MUP for staphylococci eradication in patients and medical staff, there is a risk of a significant resistance rate increase in the future. The new active compounds acting additively or synergistically with MUP can be effective in prevention of the resistance to this antibiotic.

\section{Conclusions}

Our investigation showed that FEO or tA could be added to mupirocin-based agents to eradicate the nasal carriage of S. aureus and/or to limit the emergence of mupirocin resistance as well. The FEO or tA combined with MUP tested in the study was effective against MLSB resistant $S$. aureus isolates and such combination/s may be helpful in nasal eradication of staphylococci with some patterns of resistance. This is not excluded, that in the coming future the FEO or tA will be components of agents to mucous membranes antisepsis, but more research in this field are required.

\section{Acknowledgments}

We thank the Pomeranian Medical University in Szczecin for their financial support.

\section{Conflict of interest}

The authors declare no conflict of interest.

\section{References}

1. Rongpharpi SR, Hazarika NK, Kalita H. The prevalence of nasal carriage of Staphylococcus aureus among healthcare workers at tertiary care hospital in assam with special reference of MRSA. J Clin Diagn Res 2013; 7: 257-60.

2. Boncompain CA, Suárez CA, Morbidoni HR. Staphylococcus aureus nasal carriage in health care workers: first report from a major public hospital in Argentina. Rev Argent Microbiol 2017; 49: 125-31.

3. Khanal R, Sah P, Lamichhane P, et al. Nasal carriage of methicillin resistant Staphylococcus aureus among health care workers at a tertiary care hospital in Western Nepal. Antimicrob Resist Infect Control 2015; 4: 39.

4. Coates T, Bax R, Coates A. Nasal decolonization of Staphylococcus aureus with mupirocin: strengths, weaknesses and future prospects. J Antimicrob Chemother 2009; 64: 9-15.

5. Rudresh MS, Ravi GS, Motagi A, et al. Prevalence of mupirocin resistance among staphylococci, its clinical significance and relationship to clinical use. J Lab Physicians 2015; 7 : 103-7.

6. Esquivel-Ferrińo PC, Favela-Hernández JM, Garza-González E, et al. Antimycobacterial activity of constituents from Foe- niculum vulgare var. dulce grown in Mexico. Molecules 2012; 17: 8471-82.

7. Shahat AA, Ibrahim AY, Hendawy SF, et al. Chemical composition, antimicrobial and antioxidant activities of essential oils from organically cultivated fennel cultivars. Molecules 2011; 16: 1366-77.

8. Ostad SN, Soodi M, Shariffzadeh M, et al. The effect of fennel essential oil on uterine contraction as a model for dysmenorrhea, pharmacology and toxicology study. J Ethnopharmacol 2001; 76: 299-304.

9. Ozbek H, Uğraş S, Dülger H, et al. Hepatoprotective effect of Foeniculum vulgare essential oil. Fitoterapia 2003; 74: 317-9.

10. Diao W, Hu Q, Zhang H, et al. Chemical composition, antibacterial activity and mechanism of action of essential oil from seeds of fennel (Foeniculum vulgare Mill.). Food Control 2014; 35: 109-16.

11. Shimoni E, Baasov T, Ravid U, et al. The trans-anethole degradation pathway in an Arthrobacter sp. J Biol Chem 2002; 277: 11866-72.

12. Ryu J, Seo J, Lee $Y$, et al. Identification of syn- and anti-anethole-2,3-epoxides in the metabolism of trans-anethole by the newly isolated bacterium Pseudomonas putida JYR-1. J Agric Food Chem 2005; 53: 5954-8.

13. Huang Y, Zhao J, Zhou L, et al. Antifungal activity of the essential oil of Illicium verum fruit and its main component trans-anethole. Molecules 2010; 15: 7558-69.

14. Kwiatkowski P, Mnichowska-Polanowska M, Pruss A, et al. The effect of fennel essentials oil in combination with antibiotics on Staphylococcus aureus strains isolated from carriers. Burns 2017; 43: 1544-51.

15. European Committee on Antimicrobial Susceptibility Testing (EUCAST), Breakpoints tables for interpretation of MICs and zones diameters. Version 5.0, 2015. http://www.eucast.org.

16. Clinical and Laboratory Standards Institute (CLSI), Performance standards for antimicrobial susceptibility testing. Document M100-S18, Wayne, PA (2008).

17. Saderi H, Emadi B, Owlia P. Phenotypic and genotypic study of macrolide, lincosamide and streptogramin $B\left(M_{1} S_{B}\right)$ resistance in clinical isolates of Staphylococcus aureus in Teheran, Iran. Med Sci Monit 2011; 17: 48-53.

18. Clinical and Laboratory Standards Institute (CLSI), Methods for dilution antimicrobial susceptibility test for bacteria that grow aerobically; approved standard - eight edition. Document M07-A7, Wayne, Pa (2009).

19. Krüger H, Hammer K. Chemotypes of fennel (Foeniculum vulgare Mill.). J Essent Oil Res 1999; 11: 79-82.

20. Badoc A, Deffieux G, Lamarti A, et al. Essential oil of Foeniculum vulgare Mill. (Fennel) subsp. piperitum (Ucria) Cout. fruit. J Essent Oil Res 1994; 6: 333-6.

21. Adaleti R, Nakipoglu Y, Ceran N, et al. Prevalence of phenotypic resistance of Staphylococcus aureus isolates to macrolide, lincosamide, streptogramin B, ketolid and linezolid antibiotics in Turkey. Braz J Infect Dis 2010; 14: 11-4.

22. Prabhu K, Rao S, Rao V. Inducible clindamycin resistance Staphylococcus aureus isolated from clinical samples. J Lab Physicians 2011; 3: 25-7.

23. Aydeniz OF, Cevahir N, Kaleli I. Investigation of macrolide, lincosamide and streptogramin B resistance in Staphylococcus aureus strains isolated from clinical samples by phenotypical and genotypical methods. Mikrobyol Bul 2015; 49: 1-14.

24. Nikam AP, Bhise PR, Deshmukh MM. Phenotypic detection of inducible clindamycin resistance among Staphylococcus aureus isolates. Int J Res Med Sci 2017; 5: 543-7. 
25. Yilmaz G, Aydin K, Iskender S, et al. Detection and prevalence of inducible clindamycin resistance in staphylococci. J Med Microbiol 2007; 56: 342-5.

26. Cavalcanti JM, Leal-Cardoso JH, Diniz LR, et al. The essential oil of Croton zehntneri and trans-anethole improves cutaneous wound healing. J Ethnopharmacol 2012; 144: 240-7.

27. Marinov V, Valcheva-Kuzmanova S. Review on the pharmacological activities of anethole. Scr Sci Pharm 2015; 2: 14-19.

28. Kazemi M, Mousavi E, Kharestani H. Chemical composition and antimicrobial activities of essential oils of Varthemia persica, Foeniculum vulgare, Ferula lycia. Curr Res Bacteriol 2012; 5: 42-52.

29. Lachowicz KJ, Jones GP, Briggs DR, et al. The synergistic preservative effects of the essential oils of sweet basil (Ocimum basilicum L.) against acid-tolerant food microflora. Lett Appl Microbiol 1998; 26: 209-14.

30. Itaqui SR, Verdi CM, Tondolo JS, et al. In vitro synergism between azithromycin or terbinafine and topical antimicrobial agents against Pythium insidiosum. Antimicrob Agents Chemother 2016; 60: 5023-5.

31. Sato M, Tanaka H, Yamaguchi R, et al. Synergistic effects of mupirocin and an isoflavanone isolated from Erythrina variegata on growth and recovery of methicillin-resistant Staphylococcus aureus. Int I Antimicrob Agents 2004; 24: 241-6.

32. Kifer D, Mužinić V, Klarić MŠ. Antimicrobial potency of single and combined mupirocin and monoterpenes, thymol, menthol and 1,8-cineole against Staphylococcus aureus planktonic and biofilm growth. J Antibiot (Tokyo) 2016; 69: 689-96. 